\title{
Filtration - Understanding Techniques, Types of Filters and Blocking of Filters
}

\author{
M.S. Mahadevan* and lvy Lovist
}

\begin{abstract}
The criticality of filtration in day-to-day life is most often taken for granted as the processes of filtration is shrouded in mystery, inspite of the fact that this technology has been used in one form or the other for more than 100 years. It is the purpose of this article to present the theorefical principles dealing with the technology, types of filters and the principles of blocking of filfers.
\end{abstract}

\section{Introduction}

Filtration is the most critical unit operation in daily human life as well the Industry including Pharmaceutical, Textiles, Automobile etc., The end application makes it more critical in terms of the application of this technology. Pharmaceutical and Biotechnology industries consider this as the most important unit operation as this is the final stage the life saving drug, or injectable sees before the filling takes place and the patient is administered the drug product.

Filtration is a technique used either to separate solutes from stream of solvents, remove impurities from an organic solution or to isolate an organic solid or purify molecules of varying particle size. The use of the right type of filter is fundamental

\footnotetext{
* General Manager, Biopharmaceutical Division, Millipore (India) Pvt. Limited.

† Validation Manager, Biopharmaceutical Division Millipore (India) Pvt. Limited
} 
to this separation, removal or purification process. We will delve in the basics of filtration, to unwrap the mysteries in this technology.

The two types of filtration commonly used in chemistry laboratories are gravity filtration and vacuum or suction filtration. However there is more to this technique of filtration in terms of the technology. In general terms, therefore, the process of filtration consists of passing a mixture of fluid and suspended solids through a porous medium that entraps the solids in its matrix or retains them on its surface.

A wide variety of materials have been used as filter media depending on the type of technology used in filtration. Among the more familiar are:

- porous or fibrous materials such as paper or felt;

- fabrics woven of yarns,

- monofilament plastics or fine metal wire;

- perforated or chemically etched metal sheets;

- beds of fine granular materials such as sand, charcoal or diatomaceous earth; sintered and cast powders and unglazed ceramic materials; and

- cast polymeric membranes.

Filtration can be used to meet special liquid separation requirements and the various technologies used in Filtration may be classified as

\section{- Reverse Osmosis (RO)}

Reverse osmosis is a high-pressure, energy-efficient technique for dewatering process streams, concentrating low-molecular-weight substances in solution, or puritying wastewater. It has the ability to concentrate all dissolved and suspended solids. The permeate contains a very low concentration of dissolved solids. RO is widely used in the desalination of seawater, manufacture of large volumes of antibiotics.

\section{- Nanofiltration (NF)}

Nanofittration is a special process selected when RO and UF are not the ideal choice for separation. NF can perform separation applications that are not otherwise economically feasible, such as demineralization, colour removal, and desalination. In concentration of organic solutes, suspended solids, and polyvalent ions, the permeate contains monovalent ions and low-molecular-weight organic solutions like alcohol. 


\section{- Ultrafiltration (UF)}

Ultrafiltration is a selective fractionation process utilizing pressures up to $145 \mathrm{psi}$ (10 bar). It concentrates suspended solids and solutes of different molecular weight. The permeate contains low-molecular-weight organic solutes and salts. UF is widely used in the fractionation of milk and whey, and also finds application in protein fractionation, separation, purification.

\section{- Microfiltration (MF)}

Microfiltration is a low-pressure cross-flow membrane process for separating colloidal and suspended particles in the range of 0.05-10 microns. MF is used for fermentation broth clarification and biomass clarification and recovery. Depending on the basic filtration mechanism, particle retention mode and construction characteristics, filters may be classified either as Depth filters, Surface filters or Screen/Membrane filters.

\section{Depth Filters}

These are filters that have o porous structure and when fluid passes through the filter matrix, the particles are trapped in the matrix. They have a random pore structure and a broad pore distribution. The media for flow is very large $(3-20 \mathrm{~mm})$ and the process of particle retention within the porous structure is by "entrapment \& adsorption". These filters are used to extend the life of the pre-filters. These filters have a nominal retention rating, generally less than $95 \%$, but a High Dirt Holding Capacity. This is what we all see in our daily life including the famous water filers at home.

\section{Surface Filters}

They have a better defined polymeric structure when compared to the class of Depth filters. The media is relatively thin i.e.,less than $1 \mathrm{~mm}$ and the particle retention is at or near the surface by "size exclusion and adsorption". These filters may sometimes exhibit depth properties. They are predominantly placed in the filtration train in order to extend the life of the final filter or Sterilizing grade filter. The retention efficiency is greater than $95 \%$, are nominally rated with high Dirt Holding capacity. 


\section{Membrane Filters}

They have a controlled pore structure. It can be thought of as a geometrically regular porous matrix. The media is extremely thin i.e.less than $300 \mu \mathrm{m}$. Filtration through this type of filters is considered "absolute" because anything larger than the pore size cannot pass through. Particle retention is primarily through sieving and size exclusion mechanisms. . They have the highest retention rating and they perform by retaining the particles at or near the surface. They require the highest protection in order to optimize the outputs and operation costs.

The performance of filters are dependant on various factors, some of which are as listed below:

- Types of contaminants - soft or hard

- Size of contaminants

- Number of contaminants

- Filter media characteristics

There are several theoretical models for filter blocking. The caking model describes filter cake formation through particle buildup on the surface of the filter, rather than inside the filter pores. Gradual pore blocking, is characterized by gradual, controlled blocking of pores as a function of the amount of filtrate passing through the filter. Since particle contaminants are generally smaller than the pores, particles reaching the filter surface enter the pores and gradually build up on the inner walls until the pore is completely plugged. In complete blocking, a particle that reaches the filter surface completely blocks the pore. Gradual pore blocking behavior falls between caking and gradual blocking, so particles may either block the inner walls of pores or adhere to other particles on the surface.

The plugging of the pores in the filter media may therefore be by either of the following mechanisms:

- Cake formation wherein hard non-deformable particles accumulate on the surface of the filter with a reduction in the flowrate through the filter.

- Complete pore blocking wherein soft deformable particles completely block pores with a reduction in the flowrate through the filter.

- Gradual pore blocking where both soft and hard particles block a portion of the pore gradually resulting in a gradual decrease in the flowrate through the filter 
The mechanisms of filter plugging can be described by the differential equation:

$d 2 t / d V 2=k(d t / d V n$

where, $t=$ filtration time, $V=$ cumulative filtrate volume at time $t, k=a$ constant whose dimensions are dependent on $n$.

This equation can be used as a basis to determine the optimum combination of filters to produce liquid stream of the required quality. Understanding the technologies in filtration, the types of filters and thereby optimizing filtration and utilizing the correct filter combination is the one key to successful filtration set-ups. 